\title{
Relationship between Early Inflammatory Response and Clinical Evolution of the Severe Multiorgan Failure in Mechanical Circulatory Support-Treated Patients
}

\author{
Raffaele Caruso, ${ }^{1}$ Jonica Campolo, ${ }^{1}$ Alessandro Verde, ${ }^{2}$ Luca Botta, ${ }^{2}$ \\ Lorena Cozzi, ${ }^{1}$ Marina Parolini, ${ }^{1}$ Filippo Milazzo, ${ }^{2}$ Sandra Nonini, ${ }^{2}$ Luigi Martinelli, ${ }^{2}$ \\ Roberto Paino, ${ }^{2}$ Paolo Marraccini, ${ }^{1,3}$ and Maria Frigerio ${ }^{2}$ \\ ${ }^{1}$ CNR Institute of Clinical Physiology, CardioThoracic and Vascular Department, Niguarda Cà Granda Hospital, \\ Piazza Ospedale Maggiore 3, 20162 Milan, Italy \\ ${ }^{2}$ CardioThoracic and Vascular Department, Niguarda Cà Granda Hospital, Piazza Ospedale Maggiore 3, 20162 Milan, Italy \\ ${ }^{3}$ CNR Institute of Clinical Physiology, Via Moruzzi 1, 56124 Pisa, Italy \\ Correspondence should be addressed to Raffaele Caruso; raffaele.caruso@ospedaleniguarda.it
}

Received 10 January 2014; Revised 27 June 2014; Accepted 27 June 2014; Published 14 July 2014

Academic Editor: Daqing Ma

Copyright (C) 2014 Raffaele Caruso et al. This is an open access article distributed under the Creative Commons Attribution License, which permits unrestricted use, distribution, and reproduction in any medium, provided the original work is properly cited.

Background. The mechanical circulatory support (MCS) is an effective treatment in critically ill patients with end-stage heart failure (ESHF) that, however, may cause a severe multiorgan failure syndrome (MOFS) in these subjects. The impact of altered inflammatory response, associated to MOFS, on clinical evolution of MCS postimplantation patients has not been yet clarified. Methods. Circulating cytokines, adhesion molecules, and a marker of monocyte activation (neopterin) were determined in 53 MCStreated patients, at preimplant and until 2 weeks. MOFS was evaluated by total sequential organ failure assessment score (tSOFA). Results. During MCS treatment, 32 patients experienced moderate MOFS (tSOFA < 11; A group), while 21 patients experienced severe MOFS (tSOFA $\geq 11)$ with favorable (B group) or adverse $(n=13$, C group) outcomes. At preimplant, higher values of left ventricular ejection fraction (LVEF) and estimated glomerular filtration rate (eGFR) were the only parameter independently associated with A group. In C group, during the first postoperative week, high levels of interleukin-8 (IL-8) and tumor necrosis factor (TNF)- $\alpha$, and an increase of neopterin and adhesion molecules, precede tSOFA worsening and exitus. Conclusions. The MCS patients of $\mathrm{C}$ group show an excessive release to IL- 8 and TNF- $\alpha$, and monocyte-endothelial activation after surgery, that might contribute to the unfavourable evolution of severe MOFS.

\section{Introduction}

The mechanical circulatory support (MCS) device implantation has emerged as an alternative treatment strategy in critical ill patients with advanced heart failure (HF) [1]. The MCS devices are mechanical pumps that supplement or replace the function of a damaged left ventricle in order to maintain appropriate blood flow among patients with endstage HF (ESHF). In addition to supporting circulation, MCS, in particular left ventricular assist devices, can lead to other cardiac benefits, such as improved contractility, reduction of hypertrophy, and reversal of chamber enlargement [2]. Left ventricular assist devices are mainly used as a bridge to transplant, with the aim of increasing patient survival until an appropriate organ becomes available [3].

Overall, in critically ill patients, the negative impact of chronic comorbidity on survival is primarily influenced by the degree of multiple organ failure syndrome (MOFS) or the cumulative severity of multiple comorbidities [4]. Indeed, despite advances in technology and subsequent improvements in morbidity and mortality of patients undergoing MCS placement, postimplantation MOFS, and infections 
remain the major causes of death in these patients $[5,6]$. The MOFS seems to be influenced by the degree of the immune-inflammatory response independently of the presence of infection. In MCS-treated patients, liver dysfunction was shown to be associated to the progressive release of inflammatory mediators, such as interleukin-6 (IL-6), IL8, and C-reactive protein (CRP) [7]. Moreover, platelets and monocytes activation is associated with different MCS devices suggesting a role of their interaction in the development of haematic complications [8]. However, the impact of inflammatory response on MOFS development after MCS implant still needs to be elucidated.

The aim of this study was to investigate, in MCS-treated patients, the early inflammatory signals associated to different MOFS degrees and to their clinical evolution.

\section{Materials and Methods}

2.1. Population and Study Design. We included in the study 53 patients with ESHF, not amenable to recovery by pharmacological or conventional surgical therapy, who underwent MCS, according to guideline indications for mechanical support [9].

The hemodynamic parameters, cardiac index (CI), pulmonary capillary wedge pressure (PCWP), and right atrial pressure were measured by pulmonary artery Swan-Ganz catheter. Left ventricular ejection fraction (LVEF) was quantified by transesophageal echocardiography.

We calculated the total sequential organ failure assessment score (tSOFA) according to Pätilä et al. [10] as the highest SOFA value from preimplant to two weeks after surgery. This score is a measure of MOFS development and risk of mortality in the intensive care unit after cardiac surgery. The SOFA is a six-organ (respiration, coagulation, liver, neurological, cardiovascular, and renal) dysfunction/failure score measuring multiple organ failure daily. Each organ is graded from 0 (normal) to 4 (the most abnormal), providing a daily score of 0 to 24 points.

In sedated patients, the neurological score was computed retrospectively, when sedatives were stopped, alternatively, after their temporary discontinuation. Renal function was assessed by estimated glomerular filtration rate (eGFR) using the abbreviated formula [11].

This study complied with the principles of the Declaration of Helsinki. The study protocol has been approved by the Ethics Committee of Niguarda Cà Granda Hospital (Milan, Italy) and a signed informed consent has been obtained by all participating patients.

Peripheral blood samples for biochemical assessment were collected in fasting condition preimplant and after 4 hours 1 day, 3 days, 7 days, and 14 days from MCS.

2.2. Biochemical Assessment. Plasma and serum samples were obtained after blood centrifugation at $4000 \mathrm{rpm}$ for 10 minutes.

Plasma interleukin-6 (IL-6), IL-8, IL-10, tumor necrosis factor (TNF)- $\alpha$, IL-1 receptor antagonist (IL-1ra), soluble platelet selectin (sP-selectin), and soluble intercellular adhesion molecule type 1 (sICAM-1) were measured using specific enzyme-linked immunoassays kits (R\&D Systems, Minneapolis, MN-USA for IL-6, IL-8, IL-10, IL-1ra, sPselectin, and sICAM-1; Cayman, Ann Arbor, MI-USA for TNF- $\alpha$ ) that allow an easy and accurate quantification based on antibody reaction.

Serum CRP levels were evaluated by high-sensitive immune-turbinometric assay (Roche Diagnostic $\mathrm{GmbH}$ ) which use an anti-CRP monoclonal antibody immobilized on latex particles.

Urinary neopterin levels were measured by an isocratic HPLC method, previously reported [12], and normalized by urine creatinine concentrations. Briefly, urine samples were adequately diluted with chromatographic mobile phase $\left(15 \mathrm{mM}\right.$ of $\left.\mathrm{K}_{2} \mathrm{HPO}_{4}, \mathrm{pH} 3.0\right)$. Neopterin and creatinine levels were assessed using a Kontron instrument (pump 422-S, autosampler 465) coupled to a fluorometric detector (JASCO FP-1520, $\lambda_{\text {ex }}=355 \mathrm{~nm}$ and at $\lambda_{\mathrm{em}}=450 \mathrm{~nm}$ ) for neopterin detection and to a UV-VIS detector (BIO-RAD $1706, \lambda=240 \mathrm{~nm}$ ) for creatinine determination. Neopterin and creatinine separations were performed at $50^{\circ} \mathrm{C}$ on a $5 \mu \mathrm{m}$ Discovery C18 analytical column $(250 \times 4.6 \mathrm{~mm}$ I.D., Supelco, Sigma-Aldrich) at flow rate of $0.9 \mathrm{~mL} / \mathrm{min}$.

2.3. Statistical Analysis. Data are presented as median and interquartile range (25th-75th) or frequency. Retrospectively, patients were categorized into three groups: A, B, and C, according to severity grade of MOFS and 3-month outcome. Comparison among groups was conducted by a one-way analysis of variance, or Kruskall-Wallis in case of skewed variables, with Bonferroni post hoc test for continuous variables and by a chi-square test for categorical variables. Multivariable ordinal logistic regression models were constructed to examine the effect of biochemical and clinical parameters with group of MOFS severity as the dependent variable. Results are presented as odds ratio (OR) and their 95\% confidence interval (CI). Differences across time within patient groups were assessed by nonparametric Friedman test.

A two-tailed $P$ value $<0.05$ was considered statistically significant. All statistical tests were done with SPSS version 17.0 software (SPSS, Inc., Chicago, IL, USA).

\section{Results}

Clinical data of candidates to MCS implantation and operative characteristics are described in Table 1. Patient age ranged from 22 to 72 years (median 54 (48-61)). Thirty patients had idiopathic dilated cardiomyopathy, twenty-two ischemic cardiomyopathies, and one acute myocarditis. At preimplant, 37 patients were receiving inotropes. Median tSOFA score was $5(3-7)$.

In 49 patients intrathoracic left ventricular assist devices were implanted (48 continuous flow pumps (8 DeBakey, 6 Incor, 32 HeartMateII, 2 HeartWare), and 1 pulsatileflow pump (Novacor)) while in 4 patients an extracorporeal continuous-flow, centrifugal-type rotary pump (Levitronix LLC) was implanted. 
TABLE 1: Clinical characteristics of patients and perioperative data.

\begin{tabular}{|c|c|c|c|c|c|}
\hline & \multirow{2}{*}{$\begin{array}{l}\text { All cases } \\
(n=53)\end{array}$} & \multirow{2}{*}{$\begin{array}{c}\max \text { tSOFA score }<11 \\
(\text { Group } \mathrm{A}=32)\end{array}$} & \multicolumn{2}{|c|}{$\max$ tSOFA score $\geq 11$} & \multirow{2}{*}{$P$} \\
\hline & & & Outcome $+($ group $\mathrm{B}=8)$ & Outcome $-($ group $\mathrm{C}=13)$ & \\
\hline Age, years & $54(48-61)$ & $52(45-58)$ & $53(49-62)$ & $57(52-66)$ & 0.052 \\
\hline Male gender, $n(\%)$ & $49(93)$ & $31(97)$ & $7(88)$ & $11(85)$ & 0.313 \\
\hline INTERMACS profile, $n(\%)$ & & & & & 0.610 \\
\hline 1 & $15(28)$ & $8(25)$ & $2(25)$ & $5(38)$ & \\
\hline 2 & $15(28)$ & $10(31)$ & $2(25)$ & $3(23)$ & \\
\hline 3 & $22(42)$ & $14(44)$ & $4(50)$ & $4(31)$ & \\
\hline 4 & $1(2)$ & - & - & $1(8)$ & \\
\hline Etiology, $n(\%)$ : & & & & & 0.318 \\
\hline DCM & $30(57)$ & $20(63)$ & $5(63)$ & $5(39)$ & \\
\hline $\mathrm{ICM}$ & $22(41)$ & $12(37)$ & $3(37)$ & $7(53)$ & \\
\hline Myocarditis & $1(2)$ & - & - & $1(8)$ & \\
\hline MAP, mmHg & $75(70-85)$ & $78(72-85)$ & $75(69-83)$ & $73(68-83)$ & 0.499 \\
\hline LVEF, \% & $21(18-26)$ & $23(18-26)$ & $26(19-28)$ & $20(16-21)$ & 0.045 \\
\hline LVEDV, mL & $260(190-312)$ & $260(190-330)$ & $239(174-326)$ & $237(161-292)$ & 0.466 \\
\hline $\mathrm{RAP}, \mathrm{mmHg}$ & $6(4-10)$ & $5(3-8)$ & $8(4-15)$ & $9(4-16)$ & 0.163 \\
\hline PCWP, mmHg & $24(18-30)$ & $24(15-30)$ & $23(10-33)$ & $24(19-31)$ & 0.865 \\
\hline $\mathrm{CI}, \mathrm{L} / \mathrm{min} / \mathrm{m}^{2}$ & $1.69(1.36-2.00)$ & $1.70(1.51-2.08)$ & $1.32(1.07-1.47)^{*}$ & $1.69(1.45-1.83)$ & 0.023 \\
\hline \multicolumn{6}{|l|}{ Treatments, $n(\%)$} \\
\hline ACEi + ATII & $34(67)$ & $21(68)$ & $5(63)$ & $8(67)$ & 0.961 \\
\hline Beta-blocker & $31(61)$ & $20(65)$ & $6(75)$ & $5(42)$ & 0.259 \\
\hline Statins & $13(27)$ & $9(30)$ & $2(25)$ & $2(18)$ & 0.745 \\
\hline Diuretics & $39(77)$ & $25(81)$ & $7(88)$ & $7(58)$ & 0.219 \\
\hline Inotropic & $37(71)$ & $23(72)$ & $5(63)$ & $9(75)$ & 0.824 \\
\hline Inotropic equivalents, $n$ & $5(0-10)$ & $4(0-9)$ & $4(0-8)$ & $9(1-13)$ & 0.335 \\
\hline IABP, $n(\%)$ & $22(43)$ & $13(42)$ & $2(25)$ & $7(58)$ & 0.329 \\
\hline WBC, $10^{9} / \mathrm{L}$ & $8.4(7.1-11.4)$ & $8.8(7.2-11.9)$ & $8.5(5.6-17.2)$ & $8.4(6.5-9.4)$ & 0.580 \\
\hline INR & $1.2(1.1-1.4)$ & $1.2(1.0-1.5)$ & $1.2(1.1-1.2)$ & $1.2(1.1-1.4)$ & 0.407 \\
\hline Lactate, $\mathrm{mg} / \mathrm{dL}$ & $1.0(0.8-1.8)$ & $1.2(0.8-1.7)$ & $1.0(0.7-1.8)$ & $1.0(0.8-2.8)$ & 0.814 \\
\hline $\mathrm{eGFR}, \mathrm{mL} / \mathrm{min} / 1.73 \mathrm{~m}^{2}$ & $80(58-100)$ & $86(74-116)$ & $57(50-76)^{*}$ & $59(42-76)^{*}$ & 0.001 \\
\hline $\mathrm{t}-\mathrm{Bil}, \mathrm{mg} / \mathrm{dL}$ & $1.04(0.63-1.86)$ & $0.98(0.61-1.89)$ & $1.28(0.89-2.37)$ & $0.84(0.57-1.91)$ & 0.482 \\
\hline tSOFA score, $n$ & $5(3-7)$ & $4(2-6)$ & $5(3-7)$ & $6(5-10)$ & 0.051 \\
\hline \multicolumn{6}{|l|}{ Perioperative data } \\
\hline Surgery time, min & $310(255-375)$ & $308(270-368)$ & $340(308-383)$ & $275(213-390)$ & 0.312 \\
\hline CPB time, $\min$ & $83(68-102)$ & $81(65-104)$ & $88(68-122)$ & $84(72-96)$ & 0.828 \\
\hline
\end{tabular}

Data are expressed as median and interquartile range (25th-75th) or number (percentage).

ACEi: angiotensin converting enzyme inhibitor; t-Bil: total Bilirubin; BUN: blood urea nitrogen; CI: cardiac index; CPB: cardiopulmonary bypass; DCM: dilated cardiomyopathy; ECMO: extracorporeal membrane oxygenation; eGFR: estimated glomerular filtrate rate; IABP: intra-aortic balloon pump; ICM: ischemic cardiomyopathy; INR: international normalized ratio; INTERMACS: Interagency Registry for Mechanically Assisted Circulatory Support; LVEF: left ventricular ejection fraction; LVEDV: left ventricular end-diastolic volume; MAP: mean arterial pressure; PCWP: pulmonary capillary wedge pressure; RAP: right atrial pressure; tSOFA: total sequential organ failure assessment; WBC: white blood cell count; +: positive; -: negative.

${ }^{*} P<0.05$ versus group A patients by Bonferroni post-hoc test.

During ICU stay, 21 patients (40\%) experienced postoperative maximal tSOFA score $\geq 11$, taken into account as severe MOFS associated with elevated mortality rates [13]. Among these patients, 13 (62\% of this group) died because of MOFS as primary or secondary cause of death, in a median of 13 (11-25) days. In particular, 3 patients suffered of hepatic injury, 3 suffered of intestinal ischemia, 2 showed a nonresponsive vasoplegia, 3 had respiratory failure, 1 was affected by major esophagus bleeding, and 1 was affected by disseminated intravascular coagulation. The other 32 experienced moderate MOFS with postoperative maximal tSOFA score < 11; they were all alive at 3 months after intervention.

Retrospectively, MCS-candidates were divided in 3 groups according to severity grade of post-operative MOFS and 3-month outcome. Group A: consisting of 32 patients with maximal post-operative tSOFA score $<11$ and without adverse events; group B: 8 patients with favorable outcome at 
TABle 2: Preimplant inflammatory characteristics.

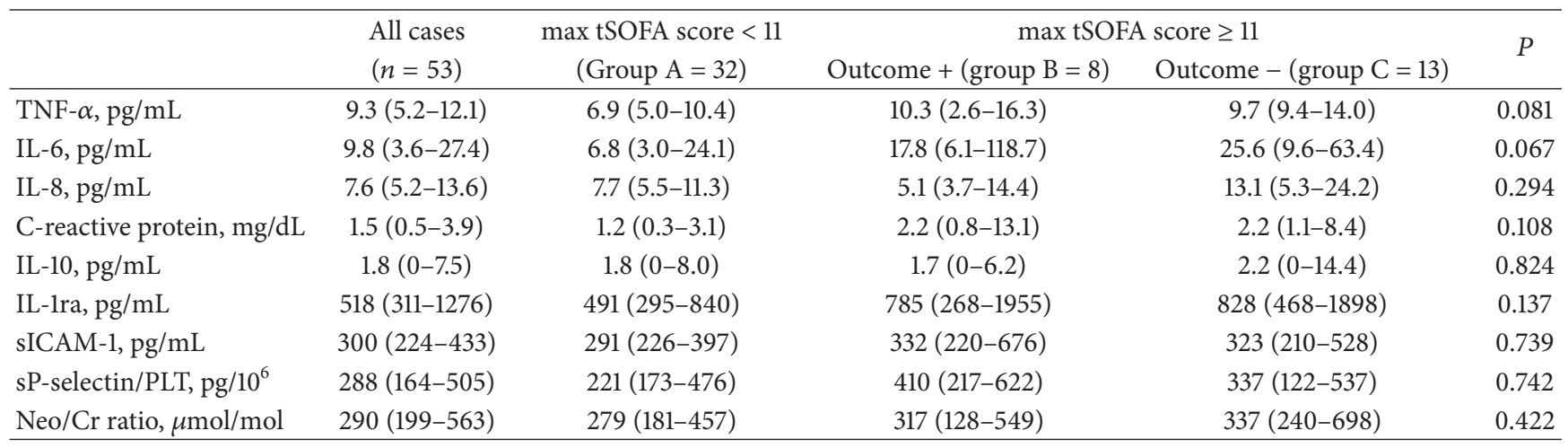

Data are expressed as median and interquartile range (25th-75th).

IL: interleukin; Neo/Cr: neopterin levels normalized by urine creatinine levels; sICAM-1: soluble form of intercellular adhesion molecule type 1; sP-selectin/PLT: soluble platelet selectin normalized by platelet count; TNF: tumor necrosis factor; +: positive; -: negative.

3 months but with maximal postoperative tSOFA score $\geq 11$; and group $\mathrm{C}$ involving the other 13 patients with and tSOFA score $\geq 11$ and adverse lethal event. Detailed pre-implant characteristics and peri-operative data of these 3 groups are described in Table 1. Age, aetiology and Interagency Registry for Mechanically Assisted Circulatory Support (INTERMACS) profiles were comparable among groups as well as the medical therapies. The LVEF and CI values are the only echocardiographic and hemodynamic parameters that differ among $\mathrm{A}, \mathrm{B}$, and $\mathrm{C}$ groups. The tSOFA score and eGFR values were different among groups, in particular eGFR levels of $B$ and $\mathrm{C}$ groups were lower than those of A group. Variables related to surgery as well as type of MCS devices used (data not shown) were comparable among groups.

3.1. Preimplant Inflammatory Status. At preimplant, the levels of proinflammatory (TNF- $\alpha$, IL-6, IL-8) and antiinflammatory (IL-10 and IL-1ra) cytokines, and adhesion molecules (sICAM-1 and sP-selectin), Neopterin/Creatinine ratios and CRP were similar among groups (Table 2).

\subsection{Preimplant Clinical Variables Associated with Moderate} MOFS. All the variables of Tables 1 and 2 that reached a $P$ value $<0.05$ were entered into the multivariable ordinal logistic regression analysis. The only variables independently associated to group A were LVEF and eGFR levels (LVEF: OR 0.847, 95\% CI 0.734-0.977, $P=0.024$; eGFR: OR 0.936, 95\% CI $0.898-0.975, P=0.001)$.

3.3. Postoperative Hemodynamic, tSOFA Score, and CRP Profiles. Postoperative recovery and hemodynamic parameters were similar among groups and were maintained during MCS.

At 1-3 postoperative days (Figure 1(a)), t-SOFA score was significantly higher in patients of $\mathrm{B}$ and $\mathrm{C}$ groups compared to A group, while at 7 and 14 days, this parameter remained higher only in $\mathrm{C}$ group compared to the others.

Serum levels of CRP were similar among group till 3 days after intervention (Figure 1(b)) but they significantly increased in B group compared to A group at 7 days postimplant. At 14 days, only patients of $\mathrm{C}$ group showed higher CRP levels with respect to A group while no difference was instead observed between $\mathrm{B}$ and $\mathrm{C}$ groups.

3.4. Postoperative Profiles of Proinflammatory Cytokines and Neopterin. At 1 postoperative day (Figure 2(a)), TNF- $\alpha$ levels significantly increased in patients of $\mathrm{C}$ group with respect to A group, while after 3 and 7 days the TNF- $\alpha$ concentrations of $\mathrm{C}$ group were higher compared to both $\mathrm{A}$ and $\mathrm{B}$ groups. IL-6 levels (Figure 2(b)) differed among groups at 7 days but also at 14 days with $\mathrm{B}$ and $\mathrm{C}$ groups having higher IL-6 concentrations than those of A group.

At 1 and 3 postoperative days, IL-8 levels (Figure 2(c)) were significantly increased in $\mathrm{C}$ group than in A group while at 7 and 14 days, the IL- 8 concentrations of $C$ group were also significantly higher compared with those of B group.

In regard to neopterin, an established marker of monocyte activation, we observed an increase of its concentration in patients of $C$ groups with respect to A group at 1, 3, and 7 days (Figure 2(d)).

No significant differences of TNF- $\alpha$, IL-8, and neopterin levels at each postoperative point were observed between $\mathrm{A}$ and $\mathrm{B}$ groups.

3.5. Postoperative Profiles of Anti-Inflammatory Cytokines. The IL-10 levels (Figure 3(a)) were significantly different in $\mathrm{C}$ group than in A group at 7 and 14 days while the IL-1ra concentrations (Figure 3(b)) were different between $\mathrm{C}$ and $\mathrm{A}$ groups of patients at $1,3,7$, and 14 days after intervention but also with $\mathrm{B}$ group at 14 days.

3.6. Postoperative Profiles of Adhesion Molecules. sP-selectin o and sICAM-1 levels (Figures 4(a) and 4(b)) were increased in patients of $\mathrm{C}$ group than in those of $\mathrm{A}$ group at 7 days post device implant.

No significant differences of sP-selectin and sICAM-1 levels were instead observed between A- and B-groups. 


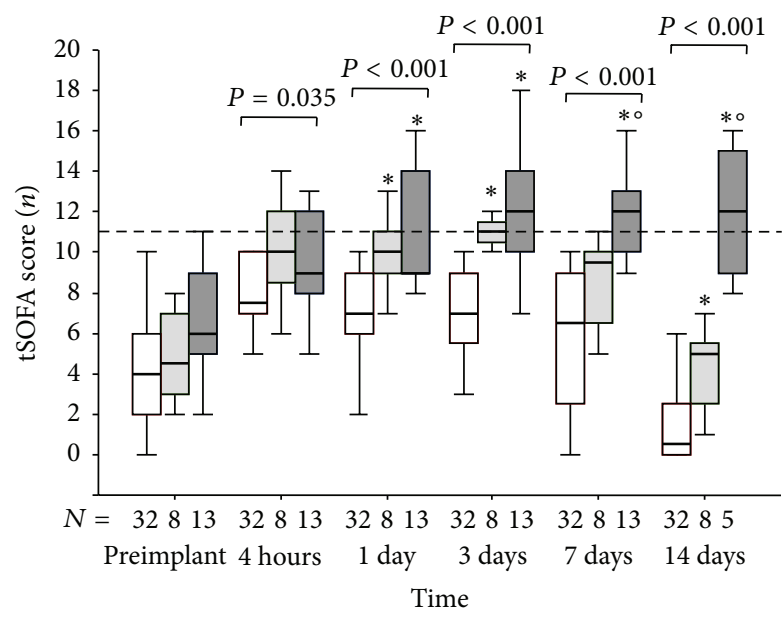

(a)

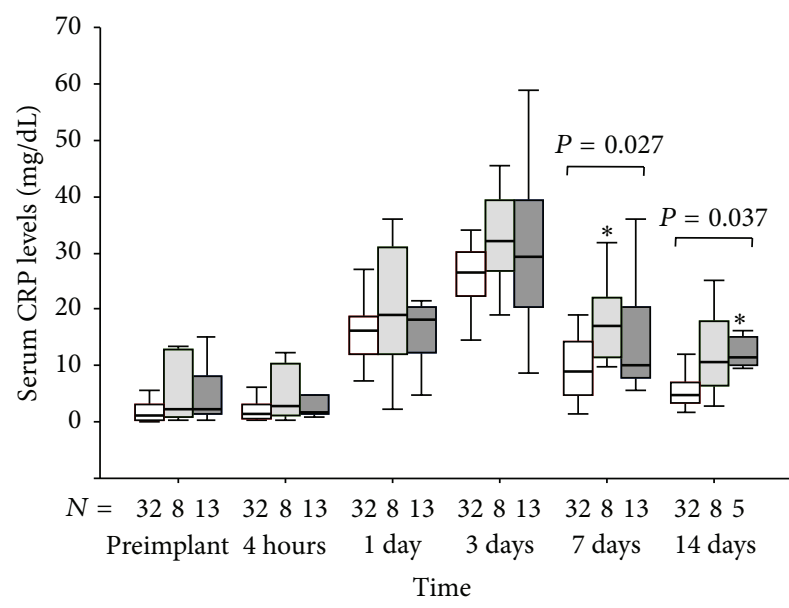

(b)

FIGURE 1: (a) Time course of the t-SOFA score and (b) CRP levels in patients that experienced postoperative maximal tSOFA score $<11$ (group A: empty box-plots), and patients with postoperative maximal tSOFA score $\geq 11$, with positive (group B: light gray box-plots) or negative (group C: dark gray box-plots) 3-month outcome. The tSOFA score $\geq 11$ is pointed out by a dashed line. $P$ values are for differences among groups at each time-point by Kruskall-Wallis text. ${ }^{*} P<0.05$ versus group A by Mann-Whitney text corrected by Bonferroni. ${ }^{\circ} P<0.05$ versus group B by Mann-Whitney text corrected by Bonferroni.

3.7. Postoperative IL-8 and TNF- $\alpha$ Exposure according to Patient Groups. The grade of patient exposure to TNF- $\alpha$ and IL-8 during the first postoperative week was measured calculating the area under the curve of respective levels of cytokines from 4 hours at 1 week (equal to 164 hours) after intervention $\left(\mathrm{AUC}_{164 \mathrm{hrs}}\right)$ by trapezoidal rule. The $\mathrm{AUC}_{164 \mathrm{hrs}}$ of TNF- $\alpha$ and IL- 8 were differently distributed among patient groups (Figure 5). In particular, the TNF- $\alpha \mathrm{AUC}_{164 \mathrm{hrs}}$ of $\mathrm{C}$ group was higher with respect to A group, while the IL-8 $\mathrm{AUC}_{164 \mathrm{hrs}}$ of $\mathrm{C}$ group was higher than those of $\mathrm{A}$ and $\mathrm{B}$ groups of patients (Figures $5(\mathrm{a})$ and $5(\mathrm{~b})$ ).

The $\mathrm{AUC}_{164 \mathrm{hrs}}$ of TNF- $\alpha$ and IL- 8 of $\mathrm{B}$ group were comparable to those of A group.

\section{Discussion}

This study investigated the clinical characteristics and inflammatory mechanisms associated to evolution of severe multiorgan dysfunction in patients affected by ESHF after MCS device implantation. The study shows that ESHF patients, with preoperative reduced LVEF and eGFR values, are more susceptible to develop a severe MOFS and thus an unfavorable outcome after MCS intervention. During the first postoperative week, the grade of inflammatory response, particularly linked to early IL- 8 and TNF- $\alpha$ exposure, is associated to the clinical evolution of patients with severe MOFS.

The application of MCS has become an effective therapeutic option for treatment of deteriorating phase of HF. However MOFS remains a frequent early complication in MCS-treated patients, and an adequate knowledge of the mechanisms involved in its onset and deterioration are still lacking. Several studies reported that, after cardiac surgery, the grade of MOFS severity developed in the first days of
ICU stay and is associated to the clinical outcome. High tSOFA scores are proposed as useful outcome predictors [13]; independently of the starting value, a tSOFA score ranging from 8 to 11 during the first 48 hours in the ICU, is associated to a mortality rate of 60 to $90 \%$. In the present study, we used an increase in $\mathrm{tSOFA}$ scores $\geq 11$ during the first weeks in ICU as threshold value to distinguish MCS patients developing a severe MOFS from those that experienced a moderate multiorgan damage (tSOFA score $<11)$. This threshold score has been able to discriminate also in our study patients at risk to unfavorable outcome. In fact, the mortality rate of subjects with tSOFA score $\geq 11$ was $62 \%$.

tSOFA value between patients of $B$ and $C$ groups was comparably high until the third day postintervention but decreased in B group at 7 and 14 days compared to $\mathrm{C}$ group, suggesting that this period may be crucial for the clinical evolution of these critical patients.

One day postintervention, the levels of cytokines, TNF- $\alpha$ and IL-8, and the urinary concentrations of neopterin significantly increased in $\mathrm{C}$ group compared to $\mathrm{A}$ and $\mathrm{B}$ groups. Moreover, during the first postoperative week, $\mathrm{C}$ group of patients showed a greater exposure to TNF- $\alpha$ and IL- 8 with respect to the other 2 groups. TNF- $\alpha$ is known to be involved in systemic inflammatory response syndrome (SIRS), which usually precedes MOFS in several ill patients [14, 15], while IL-8 is a specific monocyte attracting chemokines that modulates monocyte activation, an important condition in organ damage and haemostatic complications [16]. These data suggest that, immediately after MCS intervention, the extent of inflammatory response related mainly to TNF- $\alpha$ and IL-8 signals could influence the development and degree of MOFS severity, contributing to subsequent clinical evolution of ESHF patients. 


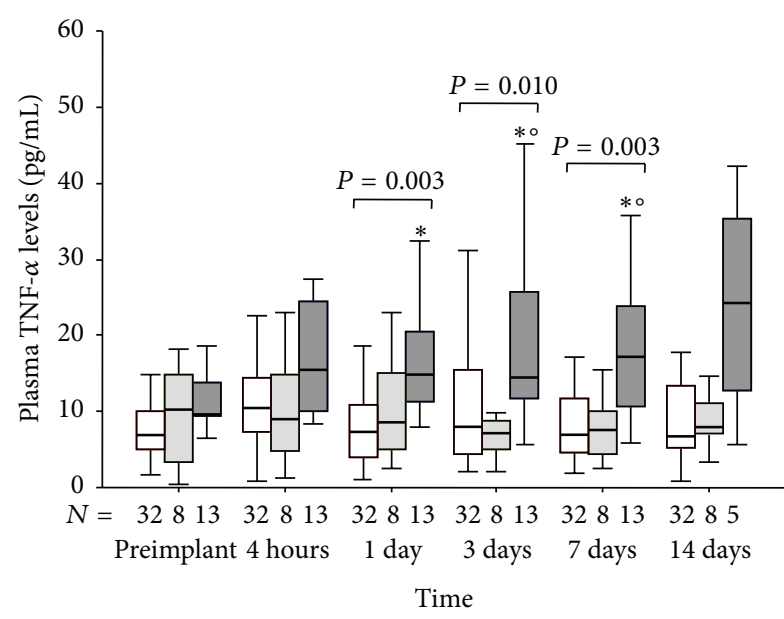

(a)

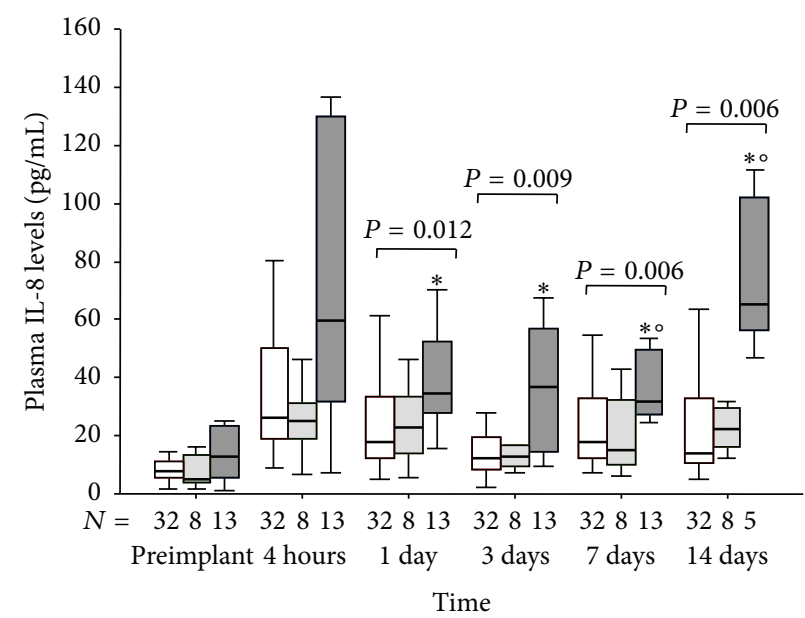

(c)

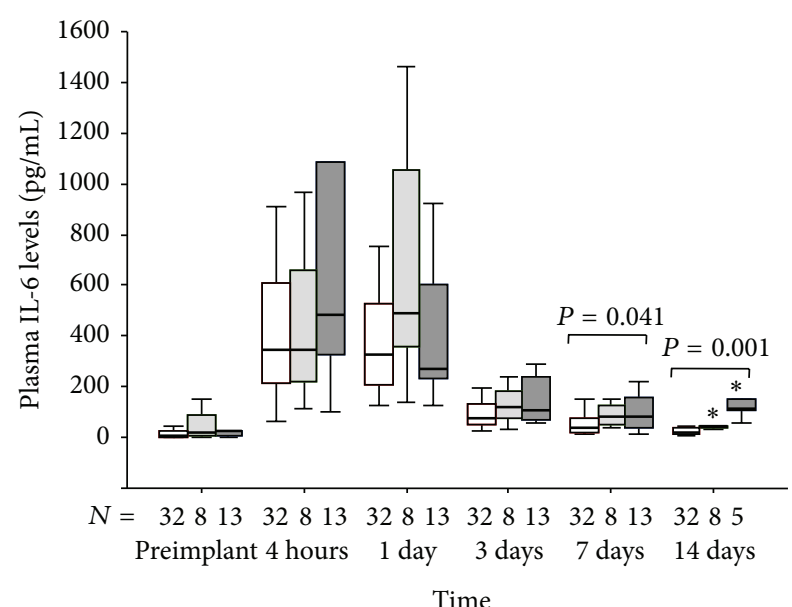

(b)

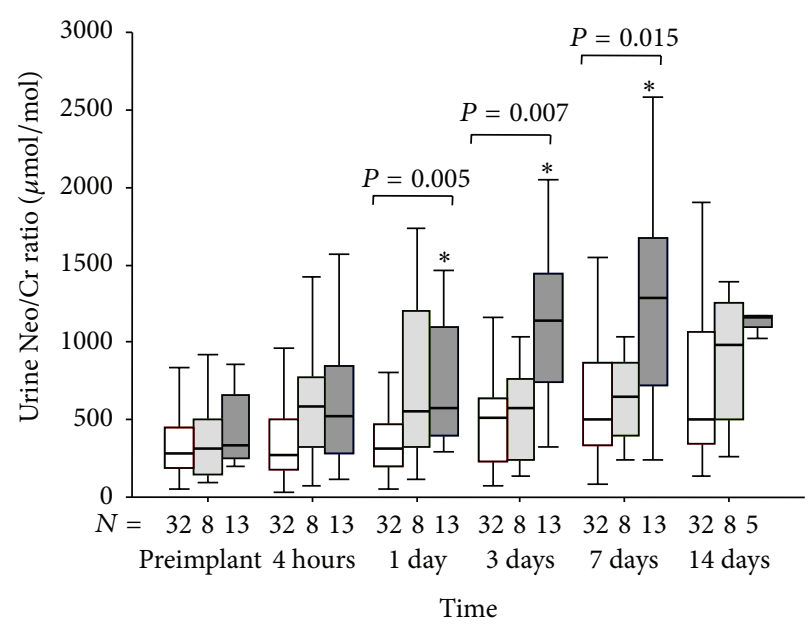

(d)

FIgURE 2: (a) Time course of plasma TNF- $\alpha$, (b) IL-6, (c) IL-8 levels, and (d) urine neopterin/creatinine ratio in patients that experienced postoperative maximal tSOFA score $<11$ (group A: empty box-plots), and patients with postoperative maximal tSOFA score $\geq 11$, with positive (group B: light gray box-plots) or negative (group C: dark gray box-plots) 3-month outcome. $P$ values are for differences among groups at each time-point by Kruskall-Wallis text. ${ }^{*} P<0.05$ versus group A by Mann-Whitney text corrected by Bonferroni. ${ }^{\circ} \mathrm{P}<0.05$ versus group $\mathrm{B}$ by Mann-Whitney text corrected by Bonferroni.

On the contrary, CRP levels, the main inflammatory variable routinely used in the setting of MCS-therapy, were not able to early discriminate patients with unfavourable outcome, suggesting that CRP profile does not reflects the inflammatory pathways involved in the adverse evolution of severe MOFS.

During the early phase of MCS, the exacerbate release of anti-inflammatory cytokines (IL-10 and IL-1ra) in addition to a massive proinflammatory reaction, could produce an unbalance of inflammatory response which might contributes to the onset of severe MOFS by causing anergy and immunosuppression [17], a condition known as immunoparalysis in patients with severe sepsis [18].

One postoperative week, patients with unfavorable evolution of severe MOFS showed also elevated sP-selectin and adhesion molecule sICAM while the neopterin levels of these patients already increased 1 day after surgery. sPselectin is a marker of platelet and endothelial alteration and a direct inducer of procoagulant activity associated with thrombotic disease $[19,20]$. sICAM is responsible for neutrophil and leukocyte attachment to the endothelium [19] while neopterin is a marker of macrophage/monocyte and immune system activation. The activation of monocyte and the injury of endothelium are factors that might contribute, in the microcirculation, to the formation of microthrombi and intravascular coagulation associated to development and worsening of multiorgan failure [7].

In previous studies, involving MCS-treated patients with left ventricular and biventricular supports, several preimplant clinical characteristics such as cardiogenic shock, advanced age, severe right heart failure, or increased bilirubine, were found to be predictors of adverse outcomes [21, 22]. In 


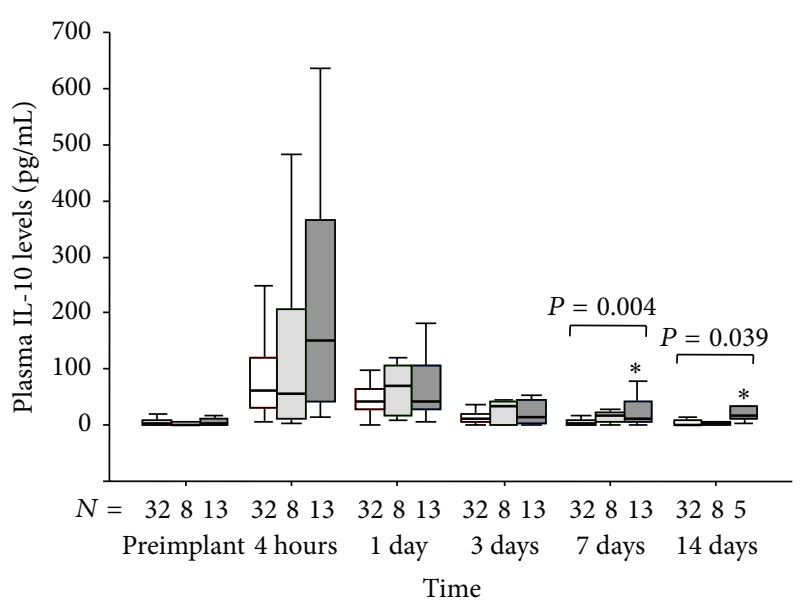

(a)

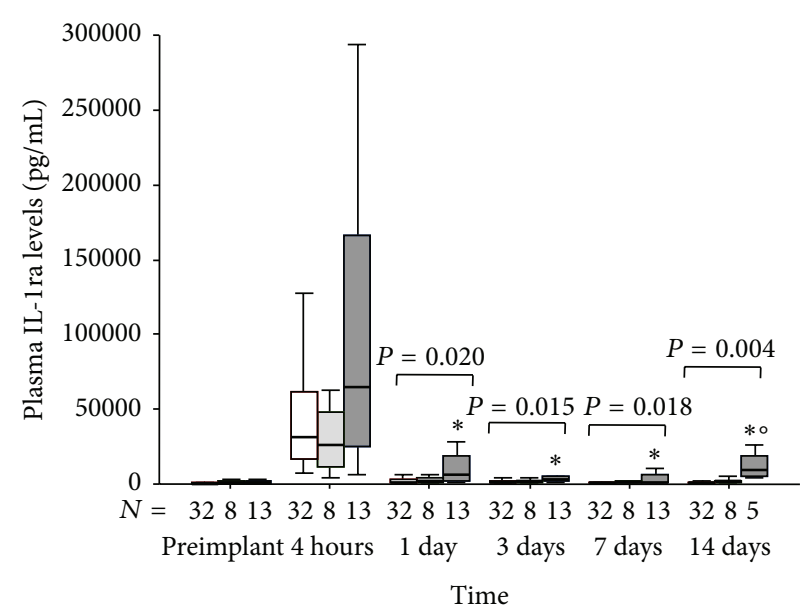

(b)

FIGURE 3: (a) Time course of plasma IL-10 and (b) IL-1ra levels in patients that experienced postoperative maximal tSOFA score < 11 (group A: empty box-plots), and patients with postoperative maximal tSOFA score $\geq 11$, with positive (group B: light gray box-plots) or negative (group C: dark gray box-plots) 3-month outcome. $P$ values are for differences among groups at each time-point by Kruskall-Wallis text. ${ }^{*} P<0.05$ versus group A by Mann-Whitney text corrected by Bonferroni. ${ }^{\circ} \mathrm{P}<0.05$ versus group B by Mann-Whitney text corrected by Bonferroni.

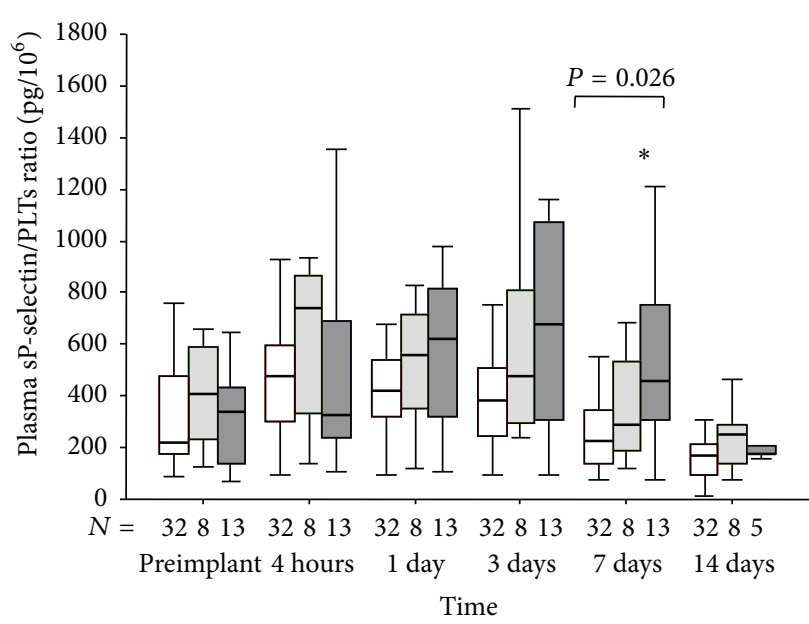

(a)

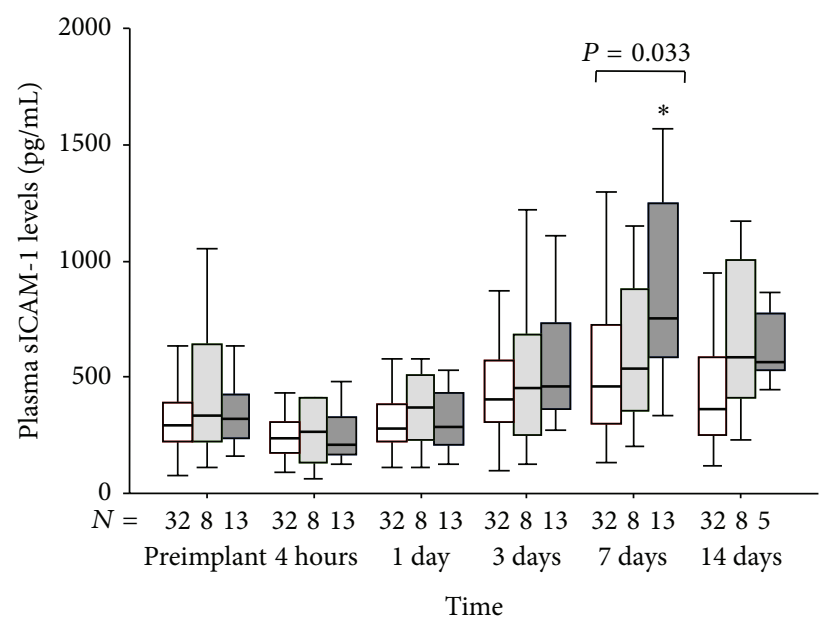

(b)

FIGURE 4: (a) Time course of plasma sP-selectin normalized by platelet (PLT) count and (b) sICAM-1 levels in patients with postoperative maximal tSOFA score < 11 (group A: empty box-plots), and patients with postoperative maximal tSOFA score $\geq 11$ and positive (group B: light gray box-plots) or negative (group C: dark gray box-plots) 3-month outcome. $P$ values are for differences among groups at each time-point by Kruskall-Wallis text. ${ }^{*} P<0.05$ versus group A by Mann-Whitney text corrected by Bonferroni. ${ }^{\circ} P<0.05$ versus group $B$ by Mann-Whitney text corrected by Bonferroni.

our series, patients characterized by lower LVEF values and altered renal function before device implantation, developed serious MOFS with unfavourable evolution after MCS intervention. On the contrary, preimplant inflammatory mediators did not differ among groups, indicating that this mechanism is activated during or immediately after surgery. The intensity and length of postimplant inflammatory response seems to be an important trigger that influences the clinical outcome of these patients.

Several studies have recently highlighted the emergent role of specialized regulatory cell subtypes belonging to myeloid-derived suppressor, monocyte phenotypes, and $\mathrm{T}$ and B lymphocytes in the proresolution of inflammatory pathways in critically ill patients $[23,24]$. The evaluation of these subtypes of inflammatory cells in subjects' candidates to MCS therapy could contribute to identify specific linkages between cellular inflammatory status and unbalanced inflammatory response associated to unfavorable outcome.

\section{Conclusions}

A specific inflammatory sequence, involving inflammatory signals dependent from TNF- $\alpha$ and IL-8, monocyte, and 


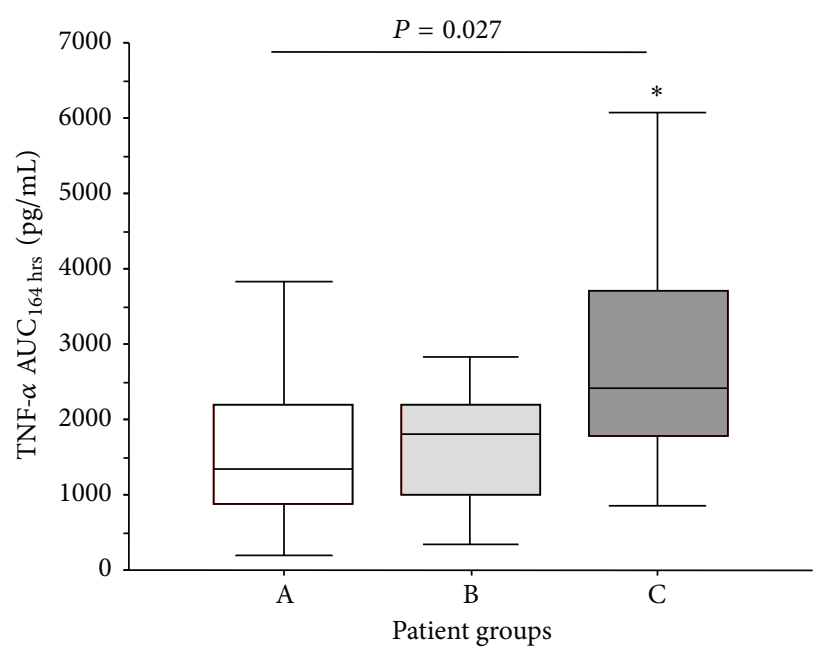

(a)

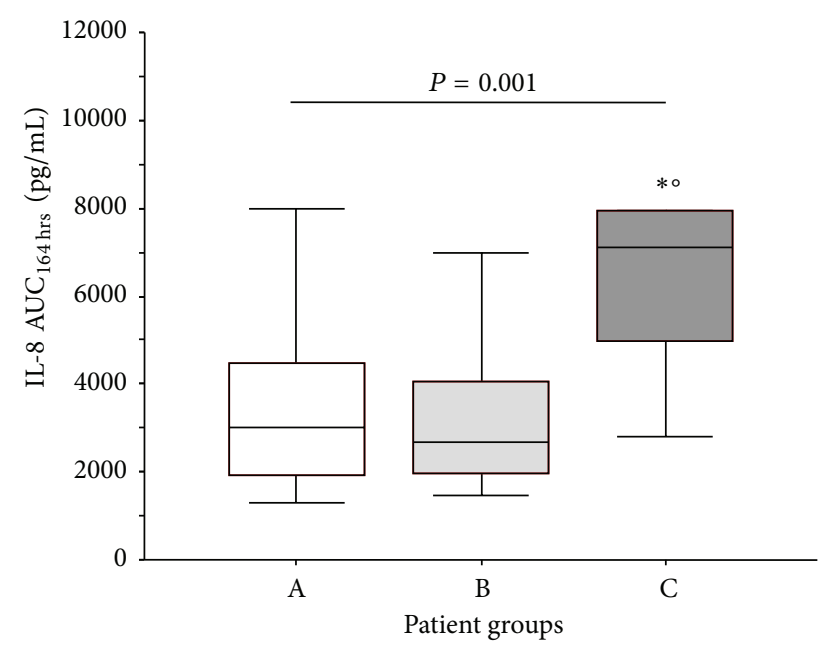

(b)

FIGURE 5: (a) The area under the curve of TNF- $\alpha$ and (b) IL-8 levels from 4 hours at 1 week after intervention (AUC 164 hrs $)_{\text {in }}$ patients that experienced postoperative maximal tSOFA score < 11 (group A: empty box-plots), and patients with postoperative maximal tSOFA score $\geq$ 11, with positive (group B: light gray box-plots) or negative (group C: dark gray box-plots) 3-month outcome. $P$ values are for differences among groups by Kruskall-Wallis text. ${ }^{*} P<0.05$ versus group A by Mann-Whitney text corrected by Bonferroni. ${ }^{\circ} P<0.05$ versus group $B$ by Mann-Whitney text corrected by Bonferroni.

endothelial activation, contributes to the unfavourable evolution of severe MOFS developed during the first weeks after MCS therapy. Patients, characterized by preimplant low ejection fraction and renal dysfunction, are more susceptible to develop an unbalanced inflammatory response during MCS therapy associated to a severe MOFS with negative outcome. High TNF- $\alpha$, IL- 8 , and neopterin concentrations may be considered as early markers of MOFS occurrence and unfavourable outcome in end stage HF patients. The correct timing to MCS implant is crucial to prevent the excessive clinical deterioration after MCS implantation triggered by an aberrant postoperative inflammatory response, which adversely affects the clinical outcome of MCS therapy.

\section{Abbreviations}

$\begin{array}{ll}\text { CRP: } & \text { C-reactive protein } \\ \text { eGFR: } & \text { Estimated glomerular filtration rate } \\ \text { ESHF: } & \text { End-stage heart failure } \\ \text { HF: } & \text { Heart failure } \\ \text { IL-: } & \text { Interleukin } \\ \text { INTERMACS: } & \text { Interagency Registry for Mechanically } \\ & \text { Assisted Circulatory Support } \\ \text { LVEF: } & \text { Left ventricular ejection fraction } \\ \text { MCS: } & \text { Mechanical circulatory support } \\ \text { MOFS: } & \text { Severe multiorgan failure syndrome } \\ \text { sICAM-1: } & \text { Soluble intercellular adhesion molecule } \\ & \text { type 1 } \\ \text { sP-selectin: } & \text { Soluble platelet selectin } \\ \text { TNF: } & \text { Tumor necrosis factor } \\ \text { tSOFA: } & \text { Total sequential organ failure } \\ & \text { assessment score. }\end{array}$

\section{Conflict of Interests}

None of the participating institutions and authors have conflict of interests regarding the study.

\section{Acknowledgments}

The authors gratefully acknowledge the skilful cooperation of the Intensive Care Unit and SC Cardiologia 2 staff of CardioThoracic and Vascular Department of Niguarda Cà Granda Hospital in Milan. This study was partially supported by FP7-ICT-2009 Project Grant agreement 24863 (SensorARTA Remote Controlled Sensorized ARTificial Heart Enabling Patients Empowerment and New Therapy Approaches).

\section{References}

[1] E. La Franca, R. Iacona, L. Ajello, A. Sansone, M. Caruso, and P. Assennato, "Heart failure and mechanical circulatory assist devices," Global Journal of Health Science, vol. 5, pp. 11-19, 2013.

[2] S. G. Drakos, A. G. Kfoury, E. H. Hammond et al., "Impact of mechanical unloading on microvasculature and associated central remodeling features of the failing human heart," Journal of the American College of Cardiology, vol. 56, no. 5, pp. 382-391, 2010.

[3] B. A. Boilson, E. Raichlin, S. J. Park, and S. S. Kushwaha, "Device therapy and cardiac transplantation for end-stage heart failure," Current Problems in Cardiology, vol. 35, no. 1, pp. 8-64, 2010.

[4] A. M. Esper and G. S. Martin, "The impact of comorbid [corrected] conditions on critical illness," Critical Care Medicine, vol. 39, no. 12, pp. 2728-2735, 2011.

[5] M. C. Deng, L. B. Edwards, M. I. Hertz et al., "Mechanical circulatory support device database of the International Society 
for Heart and Lung Transplantation: third annual report-2005," The Journal of Heart and Lung Transplantation, vol. 24, no. 9, pp. 1182-1187, 2005.

[6] S. Califano, F. D. Pagani, and P. N. Malani, "Left ventricular assist device-associated infections," Infectious Disease Clinics of North America, vol. 26, no. 1, pp. 77-87, 2012.

[7] T. Masai, Y. Sawa, S. Ohtake et al., "Hepatic dysfunction after left ventricular mechanical assist in patients with endstage heart failure: role of inflammatory response and hepatic microcirculation," Annals of Thoracic Surgery, vol. 73, no. 2, pp. 549-555, 2002.

[8] R. Radovancevic, N. Matijevic, A. W. Bracey et al., "Increased leukocyte-platelet interactions during circulatory support with left ventricular assist devices," ASAIO Journal, vol. 55, no. 5, pp. 459-464, 2009.

[9] M. S. Nieminen, M. Böhm, M. R. Cowie et al., "Executive summary of the guidelines on the diagnosis and treatment of acute heart failure: The Task Force on Acute Heart Failure of the European Society of Cardiology," European Heart Journal, vol. 26, no. 4, pp. 384-416, 2005.

[10] T. Pätilä, S. Kukkonen, A. Vento, V. Pettilä, and R. SuojarantaYlinen, "Relation of the sequential organ failure assessment score to morbidity and mortality after cardiac surgery," Annals of Thoracic Surgery, vol. 82, no. 6, pp. 2072-2078, 2006.

[11] A. S. Levey, J. P. Bosch, J. B. Lewis, T. Greene, N. Rogers, and D. Roth, "A more accurate method to estimate glomerular filtration rate from serum creatinine: a new prediction equation. Modification of Diet in Renal Disease Study Group," Annals of Internal Medicine, vol. 130, no. 6, pp. 461-470, 1999.

[12] R. Caruso, S. Trunfio, F. Milazzo et al., "Early expression of pro- and anti-inflammatory cytokines in left ventricular assist device recipients with multiple organ failure syndrome," ASAIO Journal, vol. 56, no. 4, pp. 313-318, 2010.

[13] F. Lopes Ferreira, D. Peres Bota, A. Bross, C. Mélot, and J.L. Vincent, "Serial evaluation of the SOFA score to predict outcome in critically ill patients," Journal of the American Medical Association, vol. 286, no. 14, pp. 1754-1758, 2001.

[14] F. Bishehsari, A. Sharma, K. Stello et al., "TNF-alpha gene (TNF$\alpha$ ) variants increase risk for multi-organ dysfunction syndrome (MODS) in acute pancreatitis," Pancreatology, vol. 12, no. 2, pp. 113-118, 2012.

[15] A. A. Yousef and G. A. Suliman, "The predictive prognostic values of serum TNF- $\alpha$ in comparison to SOFA score monitoring in critically ill patients," BioMed Research International, vol. 2013, Article ID 258029, 6 pages, 2013.

[16] E. Wiel, B. Vallet, and H. Ten Cate, "The endothelium in intensive care," Critical Care Clinics, vol. 21, no. 3, pp. 403-416, 2005.

[17] R. C. Bone, "Immunologic dissonance: a continuing evolution in our understanding of the systemic inflammatory response syndrome (SIRS) and the multiple organ dysfunction syndrome (MODS)," Annals of Internal Medicine, vol. 125, no. 8, pp. 680687, 1996

[18] P. Loisa, T. Rinne, S. Laine, M. Hurme, and S. Kaukinen, "Anti-inflammatory cytokine response and the development of multiple organ failure in severe sepsis," Acta Anaesthesiologica Scandinavica, vol. 47, no. 3, pp. 319-325, 2003.

[19] S. Gando, T. Kameue, N. Matsuda, M. Hayakawa, H. Hoshino, and H. Kato, "Serial changes in neutrophil-endothelial activation markers during the course of sepsis associated with disseminated intravascular coagulation," Thrombosis Research, vol. 116, no. 2, pp. 91-100, 2005.
[20] P. André, D. Hartwell, I. Hrachovinová, S. Saffaripour, and D. D. Wagner, "Pro-coagulant state resulting from high levels of soluble P-selectin in blood," Proceedings of the National Academy of Sciences of the United States of America, vol. 97, no. 25, pp. 13835-13840, 2000.

[21] W. L. Holman, R. L. Kormos, D. C. Naftel et al., "Predictors of death and transplant in patients with a mechanical circulatory support device: a multi-institutional study," Journal of Heart and Lung Transplantation, vol. 28, no. 1, pp. 44-50, 2009.

[22] A. El-Banayosy, L. Arusoglu, L. Kizner et al., "Predictors of survival in patients bridged to transplantation with the Thoratec VAD device: a single-center retrospective study on more than 100 patients," The Journal of Heart and Lung Transplantation, vol. 19, no. 10, pp. 964-968, 2000.

[23] J. N. Fullerton, A. J. O’Brien, and D. W. Gilroy, "Pathways mediating resolution of inflammation: when enough is too," Journal of Pathology, vol. 231, pp. 8-20, 2013.

[24] A. Mantovani, C. Garlanda, and M. Locati, "Macrophage diversity and polarization in atherosclerosis: a question of balance," Arteriosclerosis, Thrombosis, and Vascular Biology, vol. 29, no. 10, pp. 1419-1423, 2009. 


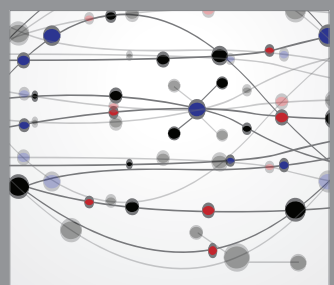

The Scientific World Journal
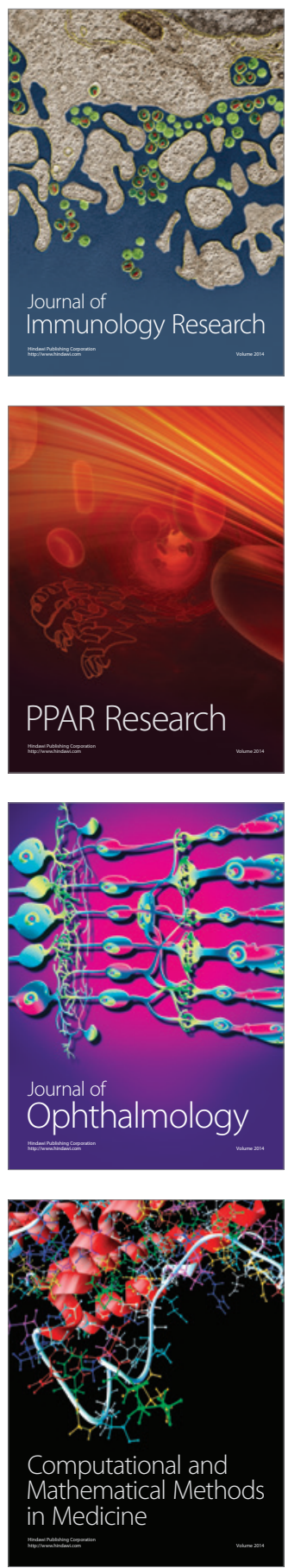

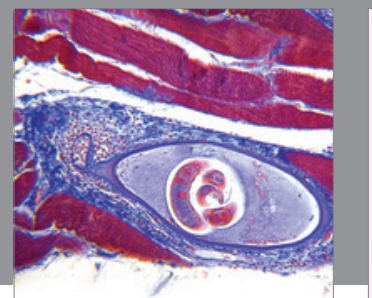

Gastroenterology

Research and Practice
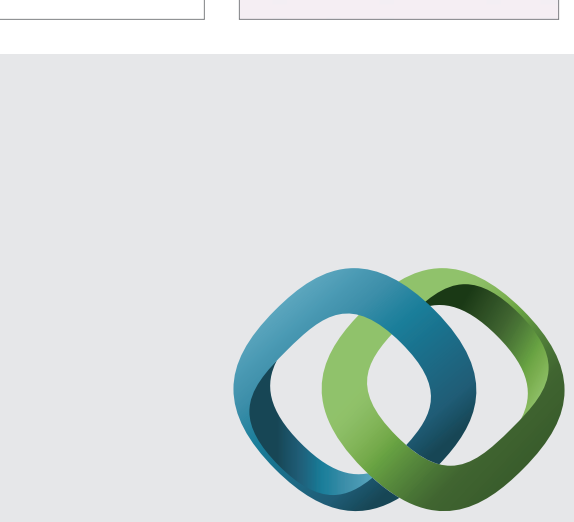

\section{Hindawi}

Submit your manuscripts at

http://www.hindawi.com
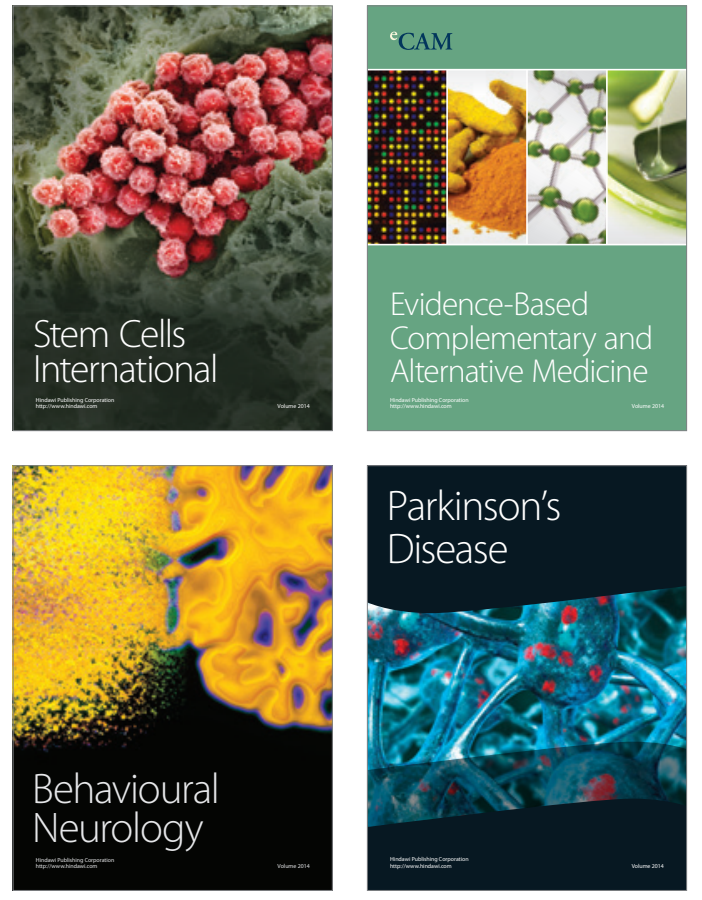
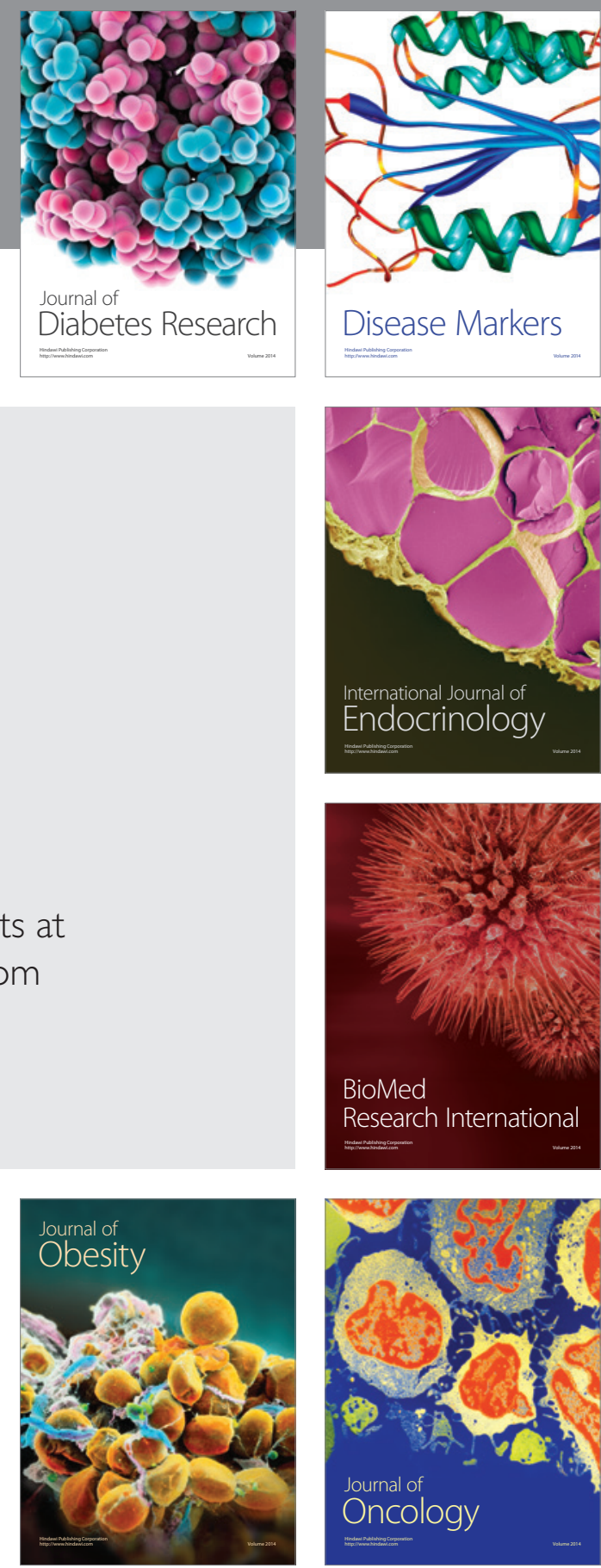

Disease Markers
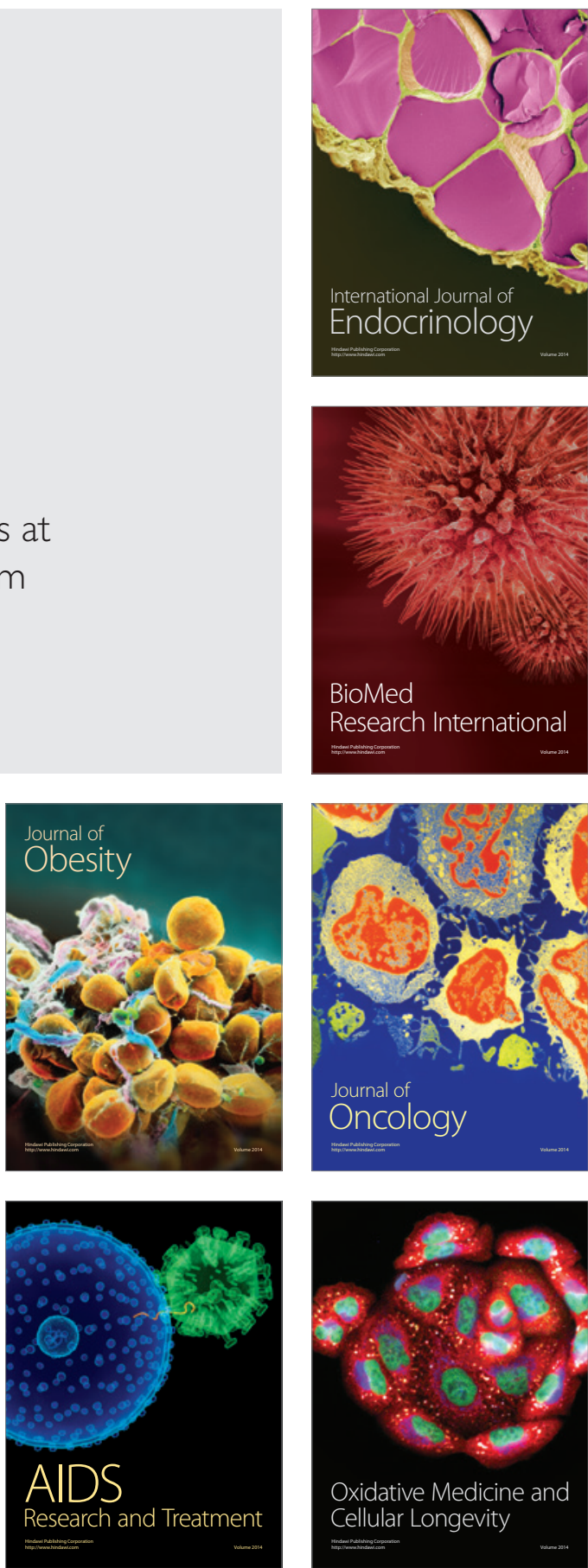